\title{
Propofol inhibits proliferation and cisplatin resistance in ovarian cancer cells through regulating the microRNA-374a/forkhead box 01 signaling axis
}

\author{
YANG SUN ${ }^{1}$, YONG-BAO PENG ${ }^{1}$, LING-LING YE ${ }^{2}$, LONG-XIAN MA ${ }^{2}$, \\ MEI-YAN ZOU ${ }^{3}$ and ZHONG-GUI CHENG ${ }^{2}$ \\ ${ }^{1}$ Department of Anesthesiology, Jiangxi Maternal and Child Health Hospital, Nanchang, Jiangxi 330003;
${ }^{2}$ Department of Anesthesiology, The First Affiliated Hospital of Nanchang University, Nanchang, Jiangxi 330006;
${ }^{3}$ Department of Obstetrics and Gynecology, Jiangxi Maternal and Child Health Hospital, Nanchang, Jiangxi 330003, P.R. China
}

Received April 8, 2019; Accepted November 18, 2019

DOI: $10.3892 / \mathrm{mmr} .2020 .10943$

\begin{abstract}
Ovarian cancer is a prominent disease that demonstrates high incidence rates in women and often presents multidrug resistance. Propofol has been demonstrated to suppress the malignancy of various types of human cancer; however, the underlying molecular mechanisms of propofol in ovarian cancer remain largely unknown. The present study aimed to investigate whether and how propofol inhibits proliferation and cisplatin (DDP) resistance in ovarian cancer cells. Ovarian cancer cell viability was assessed by the Cell Counting kit-8 assay; apoptosis and cell cycle progression were determined by flow cytometry; the relative expression levels of microRNA (miR)-374a and forkhead box O1 (FOXO1) were analyzed using reverse transcription-quantitative PCR; the binding ability of miR-374a to FOXO1 was assessed by the dual-luciferase reporter assay; cellular sensitivity to DDP was detected using the MTT assay; and finally, the protein expression levels of FOXO1, p27, and Bcl-2-like-protein 11 (Bim) were analyzed by western blotting. Propofol reduced viability, promoted apoptosis and decreased miR-374a expression levels in A2780 cells. In addition, the viability of A2780/DDP cells in the propofol + DDP treatment group was significantly inhibited, and the apoptotic rate was increased. In addition, miR-374a overexpression increased cell viability and the proportion of cells in the $\mathrm{S}$ phase, and decreased the proportion of cells in the G0/G1 phase. Conversely, genetic knockdown of miR-374a exerted the opposite effects on cell viability and cell cycle progression. Moreover, miR-374a
\end{abstract}

Correspondence to: Dr Zhong-Gui Cheng, Department of Anesthesiology, The First Affiliated Hospital of Nanchang University, 17 Yongwai Street, Donghu, Nanchang, Jiangxi 330006, P.R. China

E-mail: haohao130107@163.com

Key words: propofol, microRNA-374a, forkhead box O1, cisplatin resistance, ovarian cancer cells was demonstrated to bind to FOXO1. Propofol promoted the expression of FOXO1, p27 and Bim, induced cell cycle arrest and decreased ovarian cancer cell viability. In addition, treatment with propofol and DDP regulated FOXO1 and increased apoptosis of ovarian cancer cells. In conclusion, propofol downregulated miR-374a and modulated the FOXO1 pathway to reduce proliferation and DDP resistance in ovarian cancer cells.

\section{Introduction}

Ovarian cancer demonstrates the highest mortality rate among gynecological cancers and it is difficult to diagnose at an early stage (1). Thus, due to the majority of diagnoses occurring at advanced stages, ovarian cancer is difficult to treat and is often characterized by a low 5-year survival rate. In addition to surgical therapy, treatment of ovarian cancer often consists of chemotherapy and immunotherapy $(2,3)$. Chemotherapy is the main adjuvant therapy used for ovarian cancer, which not only affects the condition of ovarian cancer but also exerts cytotoxic activity to thoroughly eliminate cancer cells and prolong the survival time of patients. Nonetheless, resistance of cancer cells to chemotherapeutic drugs, including cisplatin (DDP), paclitaxel, vincristine and bleomycin, is a growing concern. For example, ovarian cancer cells are often resistant to paclitaxel and DDP $(4,5)$; DDP resistance is associated with an increase in DNA repair and drug inactivation, as well as changes in cellular accumulation, which prevent the efficacy of cancer treatments (6). It has been reported that STAT3 regulates microRNA ( $\mathrm{miRNA} / \mathrm{miR}$ )-216a to facilitate ovarian cancer proliferation and DDP resistance (7). Ai et al (8) reported that hypoxia-inducible factor-1 $\alpha$ (HIF-1 $\alpha)$ inhibited the response of DDP-resistant ovarian cancer cells to DDP by redirecting aerobic glycolysis towards mitochondrial oxidative phosphorylation, which promoted cellular survival through the underproduction of reactive oxygen species. Overall, this finding suggested that the HIF-1 $\alpha$-regulated cancer metabolism pathway may be a novel target for overcoming DDP resistance in ovarian cancer. Therefore, it is of importance to investigate the specific molecular mechanisms underlying DDP resistance 
in ovarian cancer to identify novel drug targets and improve the survival rate of patients with ovarian cancer.

Propofol, a central nervous system anesthetic, is often used in surgical operations in combination with inhalational anesthetics and analgesics. In addition to its function as an anesthetic, the antitumor effects of propofol have been demonstrated in gastric, lung, cervical and breast cancer (9-12). A previous study reported that propofol inhibited the invasion of ovarian cancer cells and enhanced the apoptotic effect of paclitaxel on ovarian cancer cells (13); however, the molecular mechanisms underlying these specific roles of propofol in ovarian cancer are largely unknown.

miRNAs are RNA molecules 21-23 nucleotides in length, which do not encode proteins but regulate gene expression through binding to specific miRNA-binding sites on target mRNAs $(14,15)$. miRNAs act as biological regulators in a range of cellular processes, such as cell proliferation, invasion and apoptosis or programmed cell death $(16,17)$. Notably, miR-374a negatively regulates its downstream genes to control the proliferation and invasion of cancer cells (18). Emerging evidence indicated that the transcription factor forkhead box $\mathrm{O} 1$ (FOXO1) serves important roles in controlling drug resistance in cancer cells; for example, it has been reported that FOXO1 contributes to paclitaxel-induced drug resistance in ovarian cancer (19). In addition, Wang et al (20) reported that the role of FOXO1 in paclitaxel resistance was positively regulated by thioredoxin-1 $(\operatorname{Trx} 1)$, and this effect may depend on Trx1 nuclear translocation, which was mediated by paclitaxel-induced reactive oxygen species in ovarian cancer cells. Moreover, miR-374a modulated DDP resistance in human ovarian cancer cells (21). These results suggest that the expression of miR-374a is associated with drug resistance in ovarian cancer cells; however, whether there is an interaction between propofol and miR-374a remains to be identified.

In the present study, the effect of propofol on miR-374a-induced proliferation and DDP resistance of ovarian cancer cells was investigated. It was demonstrated that propofol inhibited the growth and DDP resistance of ovarian cancer cells by decreasing miR-374a expression and consequently regulating FOXO1 expression. These findings provided a novel insight into the use of anesthetics for the treatment of ovarian cancer.

\section{Materials and methods}

Cell culture. Human ovarian cancer cell lines A2780 and DDP-resistant A2780/DDP were obtained from the American Type Culture Collection and Shanghai Enzyme Research Biotechnology Co., Ltd., respectively. Cells were cultured in DMEM supplemented with 10\% FBS (both purchased from Gibco; Thermo Fisher Scientific, Inc.) and maintained in a humidified atmosphere of $5 \% \mathrm{CO}_{2}$ at $37^{\circ} \mathrm{C}$. Upon $\mathrm{A} 2780$ or A2780/DDP cells reaching 80-90\% confluence, the cells were subcultured or inoculated for further experiments.

Drug treatment. DDP and propofol were purchased from Sigma-Aldrich; Merck KGaA. Different concentrations of propofol and DDP were used to treat ovarian cancer cell lines in a cell viability assay using different concentrations. For single agent treatment groups, ovarian cancer cells were treated with $0,1,5,10$ or $20 \mu \mathrm{g} / \mathrm{ml}$ propofol for $48 \mathrm{~h}$ at $37^{\circ} \mathrm{C}$, or
$0,5,10,20$ and $50 \mu \mathrm{M}$ DDP for $48 \mathrm{~h}$ at $37^{\circ} \mathrm{C}$. For the combined treatment group, A2780 or A2780/DDP cells were treated with $10 \mu \mathrm{g} / \mathrm{ml}$ propofol and $10 \mu \mathrm{M}$ DDP for $48 \mathrm{~h}$ at $37^{\circ} \mathrm{C}$. Cells were transfected with miR-374a or NC mimics before treatment with propofol and/or DDP.

Cell transfection. miR-374a mimics, miR-374a inhibitor and their respective negative controls (NC) with random sequences (denoted as mimics NC and inhibitor NC) were all synthesized by Shanghai GenePharma Co., Ltd. The sequences were as follows: Mimics NC forward, 5'-UUCUCCGAACGUGUC ACGUTT-3' and reverse, 5'-ACGUGACACGUUCGGAGA ATT-3'; miR-374a mimics forward, 5'-UUAUAAUACAAC CUGAUAAGUG-3' and reverse, 5'-CUUAUCAGGUUGUAU UAUAAUU-3'; inhibitors NC 5'-CAGUACUUUUGUGUAGU ACAAA-3'; miR-374a inhibitor 5'-CACUUAUCAGGUUGU AUUAUAA-3'. Full-length FOXO1 sequences were amplified and ligated into pcDNA3.1 plasmid (OriGene Technologies, Inc.) and the recombined plasmid was referred to as FOXO1. The cells were transfected with miR-374a mimics, mimics NC, miR-374a inhibitor and inhibitor NC (50 nm) using Lipofectamine ${ }^{\circledR} 2000$ reagent (Invitrogen; Thermo Fisher Scientific, Inc.), according to the manufacturer's protocol. Subsequently, the cells were treated with $10 \mu \mathrm{g} / \mathrm{ml}$ propofol and $10 \mu \mathrm{M}$ DDP treatment for $48 \mathrm{~h}$. RNA extraction and western blotting were conducted $48 \mathrm{~h}$ post-transfection.

Cell viability assay. Cell viability was measured using a Cell Counting kit-8 (CCK-8) assay (Beyotime Institute of Biotechnology) according to manufacturer's protocol. Briefly, $1 \times 10^{4}$ cells were seeded into 96 -well plates and following $10 \mu \mathrm{g} / \mathrm{ml}$ propofol treatment or transfection, $10 \mu \mathrm{l} \mathrm{CCK}-8$ solution was added into each well. Following incubation for $4 \mathrm{~h}$ at $37^{\circ} \mathrm{C}$, the absorbance was measured at $490 \mathrm{~nm}$ using a spectrophotometer. Cell viability was calculated according to the following formula: Cell viability $(\%$ of control $)=$ Experimental group (OD value)-Blank group (OD)/control group (OD)-Blank group (OD).

Flow cytometric detection of apoptosis and cell cycle progression. A total of $3 \times 10^{5}$ cells treated with propofol were cultured in 6-well plates for $24 \mathrm{~h}$. Cells were harvested using trypsin, washed twice with PBS, and subsequently subjected to an apoptosis assay with an Annexin V-FITC/PI Apoptosis Detection kit (BD Pharmingen; BD Biosciences), according to the manufacturer's protocol. Apoptotic cells were analyzed using a flow cytometry system (BD FACSCalibur; BD Biosciences) using FL1 (excitation, $488 \mathrm{~nm}$ ) and FL2 (excitation, $633 \mathrm{~nm}$ ) channels. Data were analyzed using BD CellQuest ${ }^{\mathrm{TM}}$ Pro Analysis software (BD Biosciences).

Cell cycle analysis was performed using a kit from Nanjing KeyGen Biotech Co., Ltd., according to the manufacturer's protocol. Briefly, the cells were collected and fixed with $70 \%$ ethanol at $4^{\circ} \mathrm{C}$ overnight. The cells were subsequently resuspended in PBS, and were incubated in buffer containing $50 \mu \mathrm{g} / \mathrm{ml}$ PI and $10 \mu \mathrm{g} / \mathrm{ml}$ RNase A (Beyotime Institute of Biotechnology) for $30 \mathrm{~min}$ in the dark at $37^{\circ} \mathrm{C}$. Cell cycle progression was determined using a BD LSR II flow cytometry system (BD Biosciences), and FlowJo software v10.0.7 (Tree Star, Inc.) was used for further data analysis. 
Detection of cell sensitivity to DDP using an MTT assay. To evaluate cellular sensitivity to DDP, the viability of cells treated with 5-50 $\mu \mathrm{M}$ DDP (Sigma-Aldrich; Merck KGaA) for $48 \mathrm{~h}$ was analyzed using the MTT assay (Beyotime Institute of Biotechnology). Briefly, a total of 5,000 cells were plated in 96-well plates and cultured overnight. Then, $10 \mu \mathrm{l}$ MTT solution and $100 \mu \mathrm{l}$ DMEM were added to each well for $4 \mathrm{~h}$ at $37^{\circ} \mathrm{C}$. The solution was subsequently discarded and $150 \mu \mathrm{l}$ $\mathrm{DMSO} /$ well was added to dissolve the formazan crystals. The plates were slightly agitated for $10 \mathrm{~min}$ and the absorbance was determined at $490 \mathrm{~nm}$ using a spectrophotometer. The half-maximal inhibitory concentration (IC50) values were calculated by nonlinear regression analysis using GraphPad Prism version 5.0 (GraphPad Software, Inc.) software.

Detection of caspase-3 and caspase-9 activity. Caspase-3 and caspase- 9 activities were assessed by the colorimetric method using Caspase-3 and Caspase-9 activity assay kits (Beyotime Institute of Biotechnology), respectively, according to the manufacturer's protocol. The cells were counted and washed in PBS. The samples were collected by centrifugation $\left(600 \mathrm{x} \mathrm{g} ; 5 \mathrm{~min} ; 4^{\circ} \mathrm{C}\right)$, suspended in $100 \mu 1$ lysis buffer (Beyotime Institute of Biotechnology) and kept on ice for $20 \mathrm{~min}$. Following centrifugation $\left(20,000 \mathrm{x} \mathrm{g} ; 10 \mathrm{~min} ; 4^{\circ} \mathrm{C}\right)$, the supernatant was collected for analysis. A total of $2 \mathrm{mM}$ Ac-DEVD-pNA or Ac-LEHD-pNA was used to measure caspase-3 activity or caspase- 9 activity, respectively. The absorbance was measured at $405 \mathrm{~nm}$ using a microplate reader, and caspase- 3 and caspase- 9 activity was calculated based on the relative value of $\mathrm{p}$-nitroanilide content.

Reverse transcription-quantitative PCR (RT-qPCR). Total RNA was extracted from cells using RNAiso Plus (Takara Biotechnology, Co., Ltd.). Following quantification, total RNA was reverse transcribed into cDNA using a PrimeScript RT master mix kit (Takara Biotechnology, Co., Ltd.), the reverse transcription reaction conditions were set to $37^{\circ} \mathrm{C}$ for $30 \mathrm{~min}$, and reverse transcriptase inactivation was conducted at $85^{\circ} \mathrm{C}$ for $5 \mathrm{~min}$. qPCR was subsequently performed using the SYBR ${ }^{\circledR}$ Green system (Takara Biotechnology, Co., Ltd.). The primer pairs used for the qPCR are presented in Table I. The following thermocycling conditions were used for qPCR: Pre-denaturation at $95^{\circ} \mathrm{C}$ for $10 \mathrm{~min}$, followed by a total of 40 cycles of denaturation at $95^{\circ} \mathrm{C}$ for $10 \mathrm{sec}$, annealing at $60^{\circ} \mathrm{C}$ for $50 \mathrm{sec}$ and extension at $72^{\circ} \mathrm{C}$ for $32 \mathrm{sec}$. The relative expression levels of miR-374a or FOXO1 were calculated using the $2^{-\Delta \Delta \mathrm{Cq}}$ method (22). For mRNA expression levels, $\beta$-actin was used as the internal control; for miRNA expression, U6 was used as the internal control.

Western blotting. Cells were cultured in 6-well plates, collected and total protein was extracted using RIPA lysis buffer (Beyotime Institute of Biotechnology). Total protein was quantified using a bicinchoninic acid assay kit (Bio-Rad Laboratories, Inc.) and $50 \mu \mathrm{g}$ protein samples were separated via SDS-PAGE gel on a $10 \%$ gel. The separated proteins were subsequently transferred onto nitrocellulose membranes (Merck KGaA) and blocked at room temperature for $1 \mathrm{~h}$ with $5 \%$ skimmed milk. The membranes were incubated with the following primary antibodies at $4^{\circ} \mathrm{C}$ overnight: Anti-FOXO1
Table I. Primer sequences for reverse transcription-quantitative PCR.

\begin{tabular}{ll} 
Gene & \multicolumn{1}{c}{ Primer sequence $\left(5^{\prime} \rightarrow 3^{\prime}\right)$} \\
\hline MicroRNA-374a & F: GCCGGCTTATAATACAACCTGA \\
& R: GTCGTATCCAGTGCAGGGTCCG \\
& AGGTATTCGCACTGGATACGACCA \\
& CTTA \\
FOXO1 & F: TGCATTTCGCTACCCGAGTT \\
& R: GTGGCTGACAAGACTTAACTCAA \\
GAPDH & F: CCAGGTGGTCTCCTCTGA \\
& R: GCTGTAGCCAAATCGTTGT \\
U6 & F: CTCGCTTCGGCAGCACA \\
& R: AACGCTTCACGAATTTGCGT
\end{tabular}

FOXO1, forkhead box O1; F, forward; R, reverse.

(1:1,000; ab39670; Abcam); anti-Bim (1:1,000; ab32158; Abcam); anti-p27 (1:1,000; ab32034; Abcam); and anti-GAPDH (1:2,000; ab8245; Abcam). The membranes were washed three times with TBS containing $0.1 \%$ Tween 20 . Following the primary antibody incubation, membranes were incubated with goat anti-mouse/rabbit IgG horseradish peroxidase-conjugated secondary antibodies (1:5,000; A6715/R9130; Sigma-Aldrich; Merck KGaA) for $2 \mathrm{~h}$ at room temperature. Protein bands were visualized using an ECL detection kit (Pierce; Thermo Fisher Scientific, Inc.) and protein expression was semi-quantified by ImageJ 1.52a (National Institutes of Health).

Bioinformatics analysis. Bioinformatics tools, including RAID version 2.0 software (www.rna-society.org/raid/) and miRBase (www.mirbase.org) were performed to predict the targets of miR-374a. The gene FOXO1 was identified as a potential target gene of miR-374a.

Dual-luciferase reporter assay. The dual-luciferase reporter assay was conducted as previously described (23), with a slight modification. The mutation type 3'-untranslated region (UTR) of FOXO1 gene was created by the QuikChange Site-Directed Mutagenesis kit (Stratagene; Agilent Technologies, Inc.). The wild-type or mutant (MUT) FOXO1 3'-UTR were cloned into psiCHECK ${ }^{\mathrm{TM}}-2$ vector (Promega Corporation). A total of $2 \times 10^{4}$ 293T cells (ATCC) were plated into 24-well plates and were subsequently co-transfected with wild-type or MUT plasmids (200 ng) FOXO1 3'-UTR vectors and miR-374a mimics or mimics NC using Lipofectamine ${ }^{\circledR} 2000$ (Invitrogen; Thermo Fisher Scientific, Inc.) at $37^{\circ} \mathrm{C}$ for $48 \mathrm{~h}$. The firefly luciferase activity of 293T cells was detected using the Dual-Luciferase Reporter assay system (Promega Corporation), according to the manufacturer's protocol. The firefly luciferase activity of 293T was compared with Renilla luciferase activity.

Statistical analysis. Statistical analysis of all results was performed using GraphPad Prism version 5.0 software (GraphPad Software, Inc.). Each experiment was independently conducted $\geq 3$ times and all data are presented as the 
mean \pm SD. Significant differences between groups were determined using Student's t-test or one-way ANOVA followed by Tukey's post hoc test for multiple comparisons. $\mathrm{P}<0.05$ was considered to indicate a statistically significant difference.

\section{Results}

Propofol decreases cell viability and induces apoptosis of ovarian cancer cells. To explore the effect of propofol on A2780 cells, the cell viability and apoptotic rates of A2780 cells treated with $0,1,5,10$ or $20 \mu \mathrm{g} / \mathrm{ml}$ propofol were determined. The results demonstrated that propofol exerted a significant dose-dependent inhibitory effect on cell viability and a significant dose-dependent promoting effect on the apoptotic rate of A2780 cells compared with untreated cells (Fig. 1A and B). To determine the association between the dose of propofol and miR-374a expression, RT-qPCR analysis was performed. Propofol significantly decreased the expression levels of miR-374a in A2780 cells in a dose-dependent manner compared with untreated cells (Fig. 1C). Overall, it was demonstrated that $10 \mu \mathrm{g} / \mathrm{ml}$ propofol inhibited ovarian cancer cell viability, while still maintaining moderate cell viability; however, $20 \mu \mathrm{g} / \mathrm{ml}$ led to significant cell damage compared with untreated cells (Fig. 1A). Thus, $10 \mu \mathrm{g} / \mathrm{ml}$ propofol was selected as the optimal concentration and used in subsequent experiments.

Propofol enhances DDP-induced cytotoxicity in sensitive and resistant $A 2780$ cell lines. The $\mathrm{IC}_{50}$ of DDP in resistant A2780/DDP cells was significantly higher compared with A2780 cells (40.2 vs. $14.7 \mu \mathrm{M}$; Fig. $2 \mathrm{~A})$. This result suggested that A2780/DDP cells were highly resistant to DDP. By combining the findings of previous literature and the results of the present study, a concentration near IC50 (10 $\mu \mathrm{M}$ DDP) was chosen for subsequent experiments. To investigate the effect of propofol on DDP resistance in ovarian cancer cells, an MTT assay and flow cytometric analysis were used to assess the viability and apoptosis of A2780/DDP and A2780 cells treated with DDP alone or combined with propofol. Compared with the control group, the viability of A2780 cells in the DDP-treated group was evidently reduced; but the cell viability of A2780/DDP only decreased slightly; while the viability of A2780/DDP cells in the propofol and DDP treatment group was significantly reduced compared with the control group or the DDP-only treatment group (Fig. 2B). In addition, treatment with DDP alone markedly increased the apoptosis of A2780 cells, but the apoptosis of A2780/DDP cells only increased slightly; whereas the proportion of apoptotic cells was significantly increased in A2780/DDP cells treated with propofol and DDP compared with control cells or cells treated with DDP only (Fig. 2C). Taken together, these results suggested that propofol pretreatment inhibited the activity of ovarian cancer cells, induced apoptosis and increased cell sensitivity to DDP.

Downregulation of miR-374a decreases the proliferation of ovarian cancer cells. RT-qPCR data demonstrated that propofol could downregulate the expression of miR-374a. In order to further determine the effect of miR-374a on the proliferation of A2780 cells, miR-374a was knocked down using a miR-374a inhibitor, or overexpressed using miR-374a mimics, and the proliferative ability of the cells was subsequently analyzed using the CCK-8 assay and flow cytometry. miR-374a knockdown significantly decreased miR-374a expression levels, whereas its overexpression significantly increased miR-374a expression levels compared with their respective controls (Fig. 3A), thus demonstrating successful transfection efficiency. The miR-374a mimics-transfected cells demonstrated significantly enhanced levels of cell proliferation at $72 \mathrm{~h}$ compared with the mimics NC group, whereas knockdown of miR-374a using the inhibitor significantly inhibited the proliferation of A2780 cells compared with the NC group (Fig. 3B). In addition, a significant increase or decrease in the proportion of cells in the G0/G1 phase was observed in cells transfected with the inhibitor or mimics, respectively, compared with their respective NCs (Fig. 3C). Overall, these data revealed that downregulation of miR-374a using an inhibitor blocked the cell cycle at the G0/G1 phase and inhibited the proliferation of ovarian cancer cells.

miR-374a negatively regulates FOXO1 to promote proliferation and inhibit apoptosis of ovarian cancer cells. In order to further determine the regulatory mechanism of miR-374a, the present study explored the downstream target genes of miR-374a involved in the proliferation of A2780 cells and validated the miRNA-target gene relationships. Target prediction searches were performed using RAID version 2.0, which indicated that miR-374a may directly target the 3'UTR of FOXO1 (Fig. 4A). A dual-luciferase reporter gene assay revealed that the luciferase activity of cells co-transfected with the miR-374a mimics and wild-type FOXO1 vector was significantly decreased compared with cells co-transfected with the mimics NC and wild-type FOXO1 vector (Fig. 4B). This suggested that miR-374a may bind to the 3'UTR of FOXO1. The overexpression of miR-374a in A2780 cells induced by miR-374a mimics significantly decreased FOXO1 mRNA and protein expression levels compared with the mimics NC group (Fig. 4C and D), whereas knockdown of miR-374a using the miR-374a inhibitor significantly increased the expression levels of FOXO1 at both the mRNA and protein level in A2780 cells compared with the inhibitor NC-transfected cells (Fig. 4C and D). The mRNA and protein expression levels of FOXO1 were significantly increased in cells transfected with FOXO1 compared with pcDNA3.1-transfected cells, indicating that FOXO1 was successfully overexpressed in cells following the transfection with FOXO1 (Fig. 4E). Moreover, cells with miR-374a overexpression were observed to have significantly enhanced proliferation rates and decreased apoptotic rates compared with the mimics NC group (Fig. 4F and G). miR-374a overexpression alongside FOXO1 overexpression significantly inhibited cell proliferation and increased apoptosis compared with the miR-374a mimics alone (Fig. 4F and G). Therefore, these results indicated that $\mathrm{miR}-374 \mathrm{a}$ may bind to and negatively regulate $\mathrm{FOXO1}$, facilitating the proliferation and inhibiting the apoptosis of A2780 cells.

Propofol inhibits proliferation and accelerates apoptosis of ovarian cancer cells through regulating $\mathrm{miR}-374 a / F O X O 1$. To further determine whether propofol inhibited the proliferation of ovarian cancer cells by modulating the miR-374a/FOXO1 

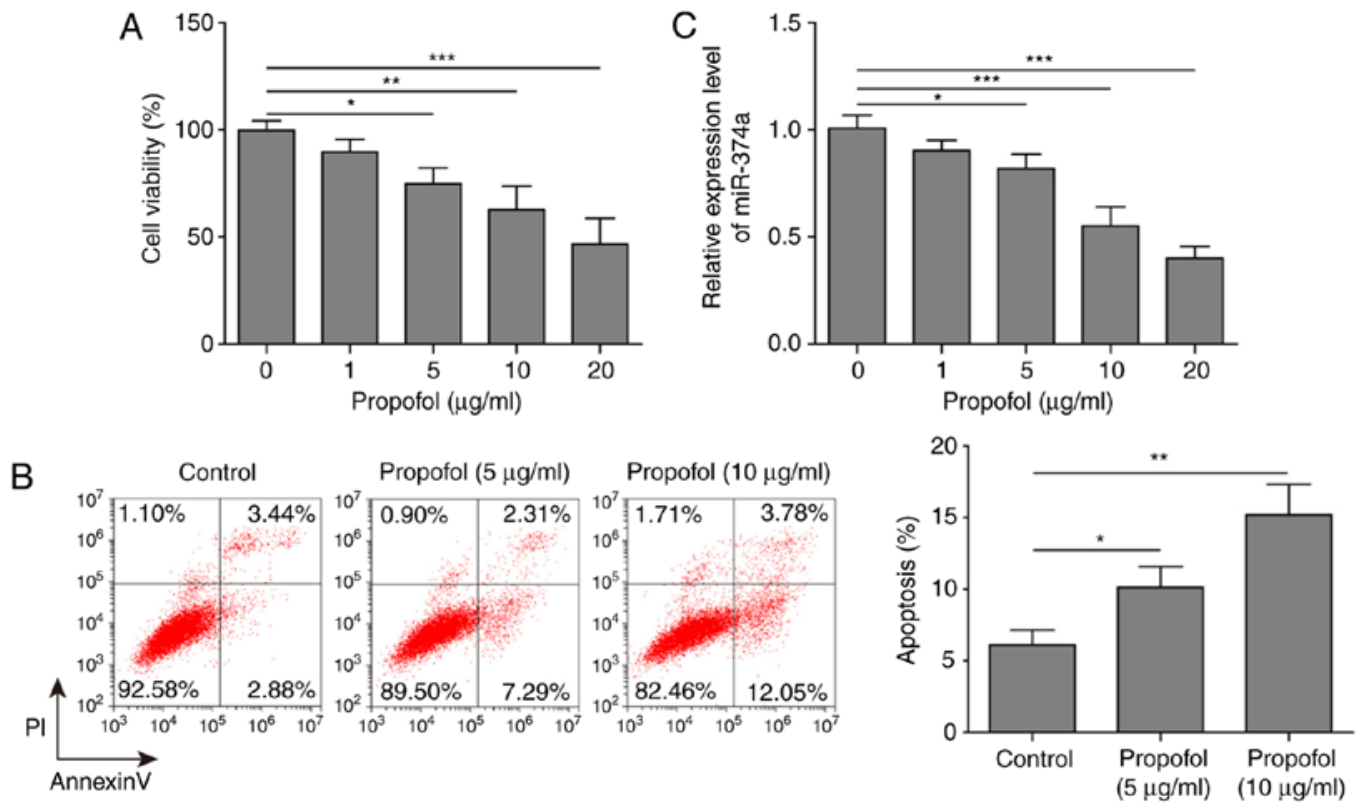

Figure 1. Propofol suppresses cell viability and promotes apoptosis of ovarian cancer cells. A2780 cells were treated with a range of propofol concentrations. (A) Cell viability was examined using a MTT assay and (B) apoptotic rate was measured using flow cytometry. (C) Relative expression levels of miR-374a were analyzed using reverse transcription-quantitative PCR. Data are presented as the mean \pm SD of three independent experimental repeats $(n=3)$. "P $<0.05$, ${ }^{* *} \mathrm{P}<0.01$ and ${ }^{* * *} \mathrm{P}<0.001$. miR, microRNA.
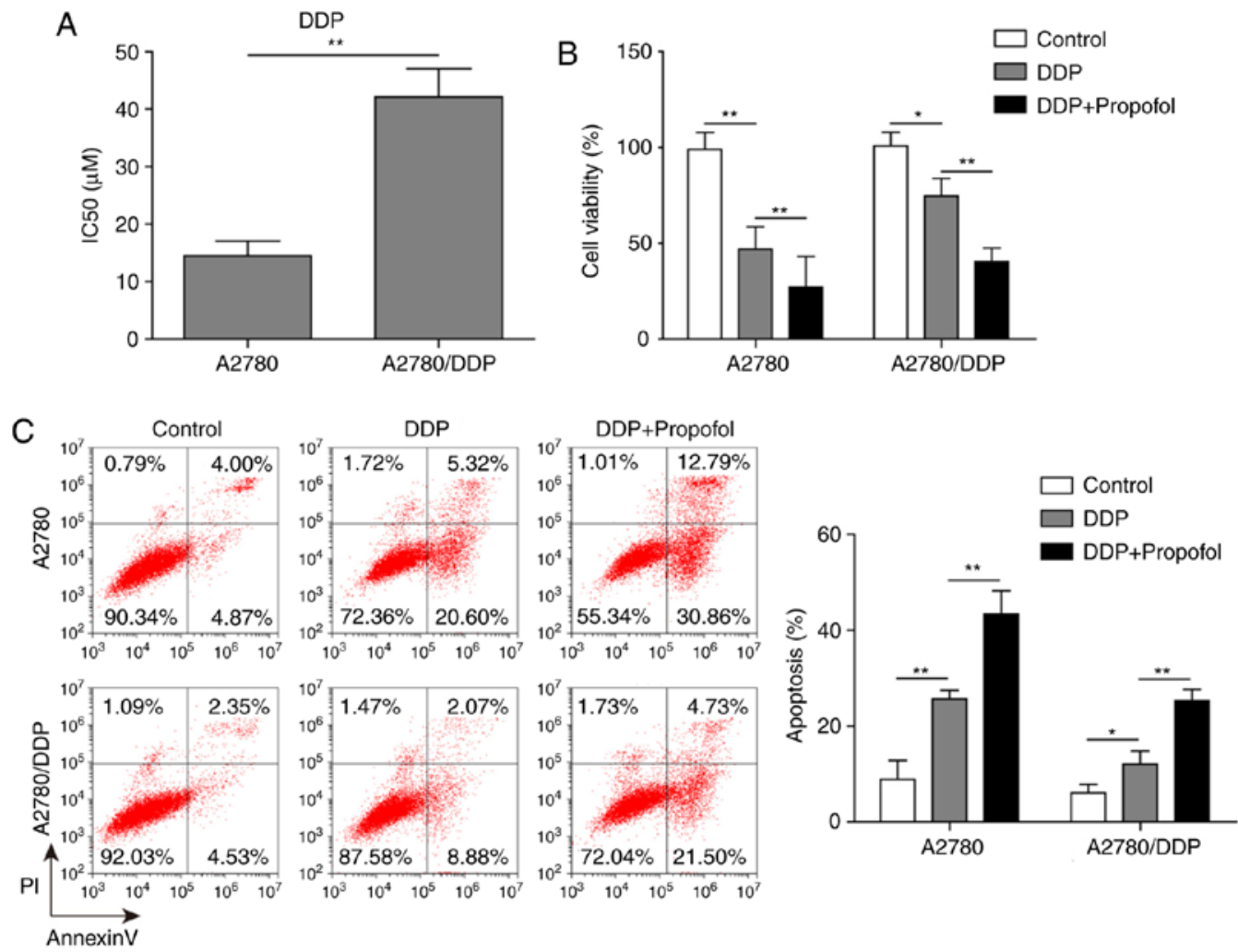

Figure 2. Propofol inhibits DDP resistance in ovarian cancer cells. (A) A2780 and DDP-resistant A2780/DDP cells were treated with DDP and the IC s0 $_{50}$ calculated using an MTT assay to validate the sensitivity of A2780 and A2780/DDP cells to DDP. (B) A2780 and A2780/DDP cells were incubated with DDP alone or DDP combined with propofol, and cell viability was analyzed using an MTT assay. (C) Apoptotic rate of A2780 and A2780/DDP cells treated with DDP or DDP in combination with propofol was measured using flow cytometry. Data are presented as the mean \pm SD, $n=3$. " $\mathrm{P}<0.05$ and ${ }^{* *} \mathrm{P}<0.01$. DDP, cisplatin.

signaling axis, the effects of propofol alone or combined with miR-374a overexpression on the cell viability, cell cycle progression and expression of proteins related to FOXO1 signaling were analyzed. Cell viability was significantly reduced in the propofol treatment group compared with the control group, whereas viability was significantly enhanced 

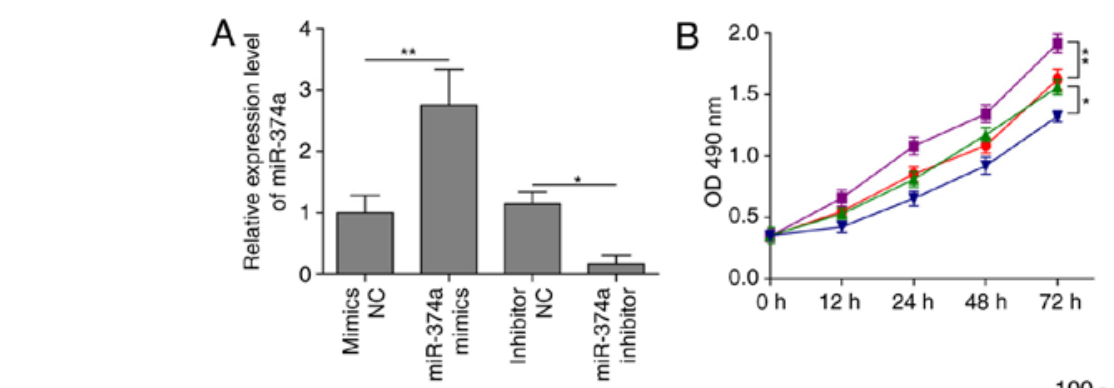

$\rightarrow$ Mimics NC

- miR-374a mimics

- Inhibitor NC

- miR-374a inhibitor
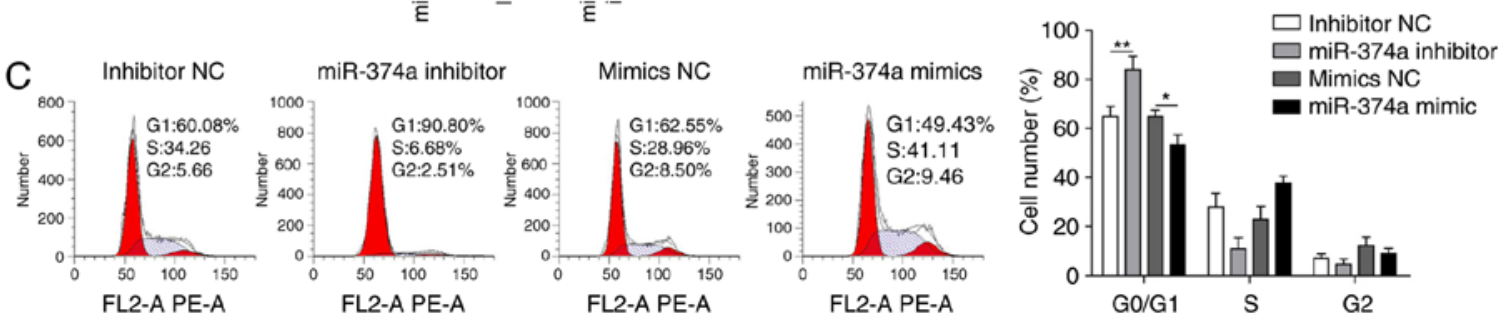

Figure 3. Knockdown of miR-374a with a miR-374a inhibitor decreases the proliferation of ovarian cancer cells. A2780 cells were transfected with mimics NC, miR-374a mimics, inhibitor NC or miR-374a inhibitor. (A) miR-374a expression levels were analyzed using reverse-transcription-quantitative PCR. (B) Cell viability was assessed using the Cell Counting kit- 8 assay. (C) Cell cycle progression was examined using flow cytometry. Data are presented as the mean \pm SD, $\mathrm{n}=3 .{ }^{*} \mathrm{P}<0.05$ and ${ }^{* *} \mathrm{P}<0.01$. miR, microRNA; NC, negative control; OD, optical density.

A

$$
\begin{aligned}
& \text { FOXO1 3'UTR WT 5'...ATTAAAAGCATATTGTATT-ATAG. } \\
& \text { Hsa-mir-374a 3GUGAaUaguccaAcauaAuaUus } \\
& \text { FOXO13 } 3^{\prime} \text { UTR MUT } \quad 5^{\prime} \text { '..ATTAAAAGCATATTGATAATATG... } 3^{\prime}
\end{aligned}
$$

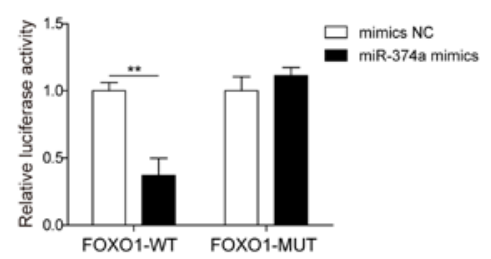

C

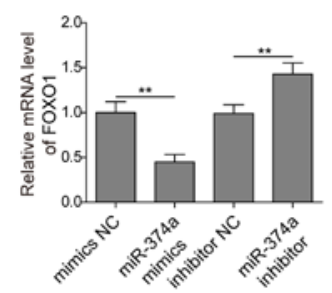

$\mathrm{E}$

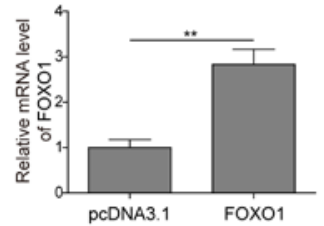

G

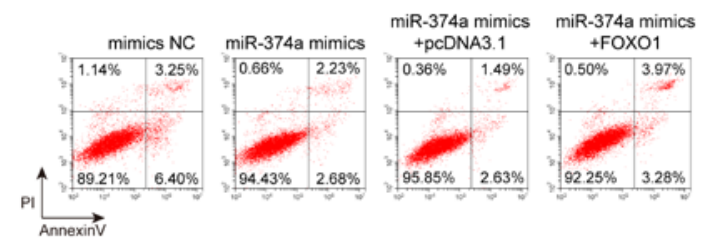

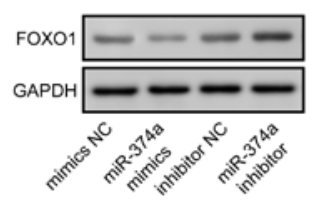
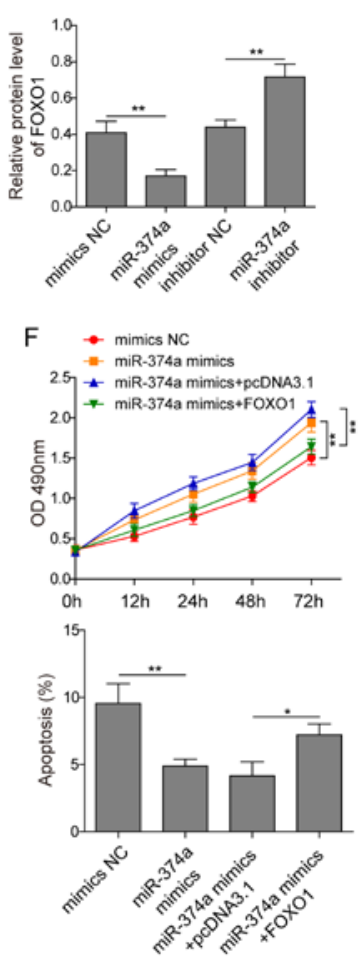

Figure 4. miR-374a negatively regulates FOXO1 to promote the proliferation and induce the apoptosis of ovarian cancer cells. (A) A potential binding site of miR-374a in FOXO1 was predicted. (B) A2780 cells were co-transfected with miR-374a mimics or mimics NC and luciferase reporter plasmids carrying WT or MUT FOXO1 3'UTR, and luciferase activity was measured. (C and D) A2780 cells were transfected with mimics NC, miR-374a mimics, inhibitor NC or miR-374a inhibitor and the relative (C) gene and (D) protein expression levels of FOXO1 were analyzed using RT-qPCR and western blotting, respectively. (E) A2780 cells were transfected with empty pcDNA3.1 plasmid or FOXO1 plasmid, and the relative gene and protein expression levels of FOXO1 were analyzed using RT-qPCR and western blotting, respectively. (F and G) A2780 cells were transfected with mimics NC, miR-374a mimics, miR-374a mimics and pcDNA3.1, or miR-374a mimics and FOXO1. (F) Cell proliferation was analyzed using a Cell Counting kit-8 assay. (G) Apoptosis was detected using flow cytometry. Data are presented as the mean $\pm \mathrm{SD}, \mathrm{n}=3$. ${ }^{*} \mathrm{P}<0.05$ and ${ }^{* *} \mathrm{P}<0.01$. FOXO1, forkhead box O1; miR, microRNA; MUT, mutant; NC, negative control; RT-qPCR, reverse transcription-quantitative PCR; WT, wild-type. 

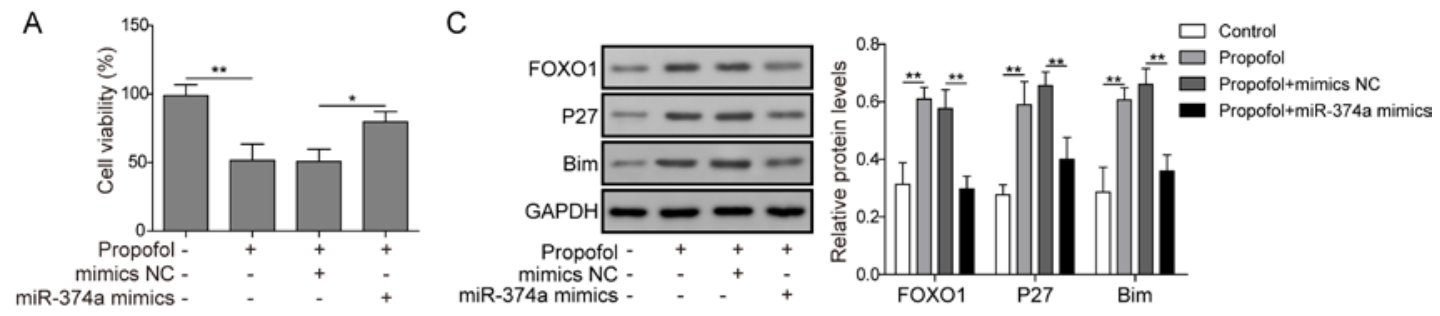

B
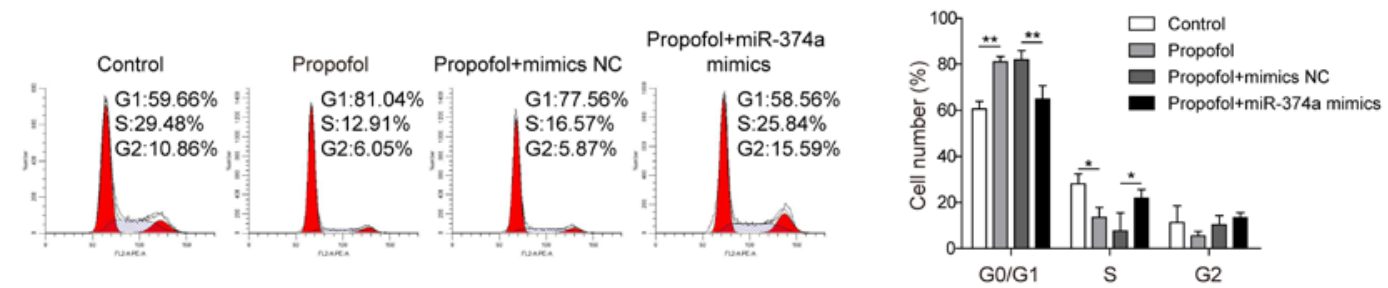

D
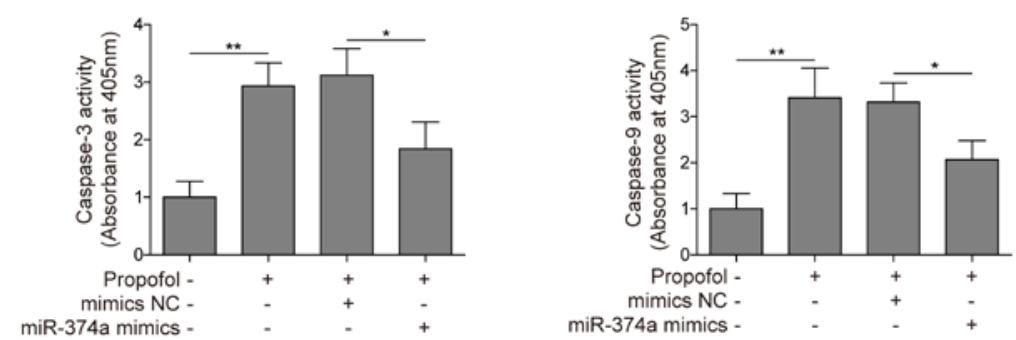

Figure 5. Propofol inhibits the proliferation of ovarian cancer cells through regulating the miR-374a/FOXO1 signaling axis. A2780 cells were transfected with mimics NC or miR-374a mimics and treated with propofol. (A) Cell viability was analyzed using the Cell-Counting kit-8 assay. (B) Cell cycle analysis was performed using flow cytometry. (C) Protein expression levels of FOXO1, p27 and Bim were determined and semi-quantified using western blotting. (D) Caspase-3 and caspase-9 activities were detected using colorimetric kits. Data are presented as the mean $\pm \mathrm{SD}, \mathrm{n}=3$. ${ }^{*} \mathrm{P}<0.05$ and ${ }^{* * *} \mathrm{P}<0.01 \mathrm{Bim}, \mathrm{Bcl}-2-\mathrm{like}$ protein 11; FOXO1, forkhead box O1; miR, microRNA; NC, negative control.

A

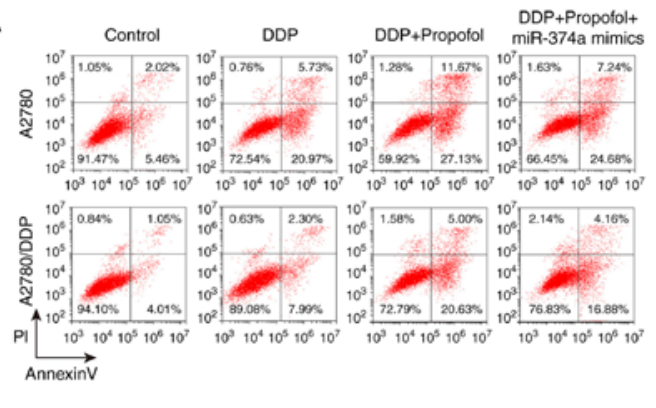
AnnexinV

B

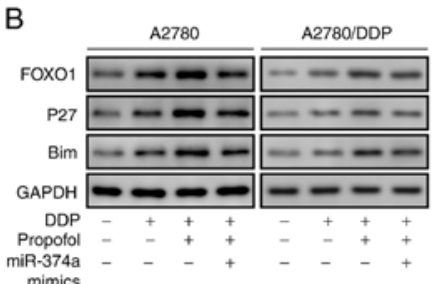

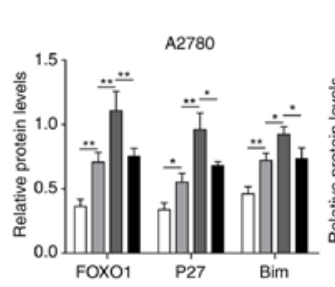
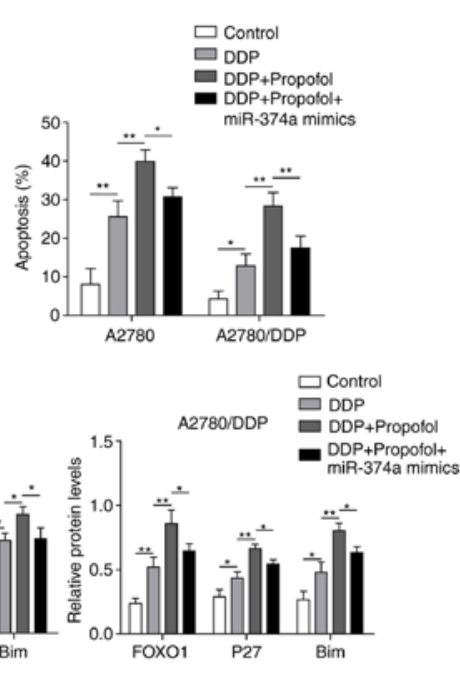

Figure 6. Propofol inhibits DDP resistance in ovarian cancer cells by regulating the miR-374a/FOXO1 signaling axis. A2780 and A2780/DDP cells were treated with DDP alone, with DDP in combination with propofol, or with DDP, propofol and miR-374a mimics. (A) Apoptotic rate of A2780 and A2780/DDP cells was assessed and semi-quantified using flow cytometry. (B) Protein expression levels of FOXO1, p27 and Bim in A2780 and A2780/DDP cells were analyzed and semi-quantified using western blotting. Data are presented as the mean $\pm \mathrm{SD}, \mathrm{n}=3$. ${ }^{*} \mathrm{P}<0.05$ and ${ }^{* *} \mathrm{P}<0.01$. Bim, Bcl-2-like protein 11; FOXO1, forkhead box O1; miR, microRNA; DDP, cisplatin.

in the propofol treatment and miR-374a overexpression group compared with the propofol treatment and mimics NC group (Fig. 5A). Furthermore, propofol significantly increased the proportion of cells in the G0/G1 phase of the cell cycle compared with the control group and decreased the proportion of cells in the $\mathrm{S}$ phase (Fig. 5B), whereas propofol combined with miR-374a overexpression significantly decreased the proportion of G0/G1 phase cells compared with the propofol and mimics NC-treated cells and increased the proportion of $\mathrm{S}$ phase cells (Fig. 5B). p27 is a CDK inhibitor that negatively regulates cell cycle progression (24) and Bim is a pro-apoptotic protein, the dysregulation of which is closely related to tumorigenesis and drug resistance (25). Western blotting was used to analyze the protein expression levels of FOXO1, p27 
and Bim. Following propofol treatment, the expression levels of FOXO1, p27 and Bim in cells were significantly increased compared with the control (Fig. 5C). However, following treatment with propofol and the overexpression of miR-374a, the expression levels of FOXO1, p27 and Bim in cells were significantly decreased compared with cells treated with propofol and mimics NC (Fig. 5C). Propofol significantly increased caspase-3 and caspase-9 activities in cells compared with untreated cells (Fig. 5D), whereas propofol combined with miR-374a overexpression significantly decreased caspase-3 and caspase- 9 activities compared with cells treated with propofol and mimics NC (Fig. 5D). Taken together, these data further demonstrated that propofol suppressed proliferation and accelerated apoptosis of ovarian cancer cells via regulation of the miR-374a/FOXO1 signaling axis.

Propofol inhibits DDP resistance in ovarian cancer cells by regulating the miR-374a/FOXO1 signaling axis. The molecular signaling mechanisms involved in the effect of propofol on the drug sensitivity of ovarian cancer cells were subsequently investigated. Following treatment with propofol and DDP, the apoptotic rate of A2780 and A2780/DDP cells was significantly increased compared with DDP only-treated cells; however, this was significantly reduced following treatment of cells with propofol, DDP and the miR-374a mimics (Fig. 6A). The protein expression levels of FOXO1, p27 and Bim in A2780 and A2780/DDP cells were significantly increased following DDP and propofol pretreatment compared with cells treated with DDP alone, but were significantly decreased following simultaneous treatment with propofol, DDP and miR-374a mimics (Fig. 6B). Overall, these data revealed that propofol may exert a positive effect by increasing the sensitivity of ovarian cancer cells to DDP by inhibiting the expression of miR-374a and subsequently promoting FOXO1 signaling.

\section{Discussion}

Previous studies have demonstrated that ovarian cancer cells can acquire DDP resistance $(26,27)$; drug resistance reduces the sensitivity of ovarian cancer cells to chemotherapeutic drugs, promotes the proliferation and metastasis of cancer cells, and shortens the survival time of patients (28). Thus, a detailed study of the mechanisms underlying drug resistance in ovarian cancer is required to provide a deeper understanding of the inhibition of ovarian cancer cells.

Propofol is an intravenous anesthetic that is mainly used for the induction and maintenance of general anesthesia during surgery $(29,30)$. However, it has been reported that propofol may inhibit the proliferation of cancer cells. Zhang et al (31) demonstrated that propofol promoted the apoptosis of cervical cancer cells by regulating the mTOR signaling pathway. Liu et al (32) also concluded that propofol suppressed the growth, migration and invasion of hepatocellular carcinoma cells by downregulating miRNA expression. Propofol enhanced the apoptotic effect of DDP on cervical cancer, which subsequently increased the sensitivity of cancer cells to the chemotherapeutic effect of DDP (33), whereas another study reported that propofol altered the resistance of prostate cancer cells to docetaxel by inducing hypoxia (34). In addition, Huang et al (35) observed that propofol inhibited the growth and invasive ability of ovarian cancer cells by modulating the miR-9/NF-kB signaling pathway. The results of the present study suggested that propofol alone inhibited the growth of ovarian cancer cells and enhanced apoptosis; however, the mechanisms through which propofol induced apoptosis and inhibited the proliferation of cancer cells are not well understood. This study demonstrated that the expression levels of miR-374a were significantly decreased by propofol in a dose-dependent manner. It was further observed that overexpression of miR-374a reversed propofol-induced cell death and the apoptosis of ovarian cancer cells, suggesting that miR-374a may serve a crucial role in propofol-induced antitumor effects. DDP is a common chemotherapeutic drug used in the clinic, which can induce cytotoxicity or damage ovarian cancer cells; however, owing to the increased usage of chemotherapeutic drugs, cancer cells mutate and exhibit reduced sensitivity towards DDP (36). In the present study, it was discovered that combining propofol with DDP reduced the viability of DDP-resistant ovarian cancer cells and promoted apoptosis, demonstrating an overall effect similar to that seen of each drug alone. Therefore, this suggested that propofol and DDP may exhibit an additive effect on the cytotoxicity over ovarian cancer cells.

miR-374a serves an important physiological role in drug resistance and proliferation of cancer cells; Li et al (21) discovered that miR-374a is a DDP-resistant regulator in human ovarian cancer cells and the expression levels of miR-374a in DDP-resistant ovarian cancer cells were higher than those in non-DDP-resistant ovarian cancer cells (19). FOXO1, a vital transcription factor that mainly participates in cell proliferation, is closely associated with drug resistance of cancer cells (37). In the present study, knockdown of miR-374a using a miR-374a inhibitor inhibited the growth of ovarian cancer cells and promoted cell cycle arrest at the G0/G1 phase. In addition, miR-374a was observed to bind to FOXO1 and negatively regulate FOXO1 expression. He et al (38) demonstrated that in human osteosarcoma, miR-374a enhanced cell growth by inhibiting downstream FOXO1 signaling, which is consistent with the results of the current study in human ovarian cancer cells. Furthermore, p27 is a CDK inhibitor that has a negative regulatory effect on proliferation and is a downstream target of FOXO1; consequently, FOXO1 inhibits cell proliferation by upregulating p27 (39-42). The Bim protein belongs to the Bcl-2 protein family and exerts a pro-apoptotic effect on cells $(43,44)$; previous studies have confirmed that FOXO1 regulates cell apoptosis through its downstream targets, including Bim $(45,46)$. The present study confirmed that propofol may regulate miR-374a/FOXO1 signaling to promote the expression of p27 and $\mathrm{Bim}$, and to inhibit the proliferation and promote the apoptosis of ovarian cancer cells, thereby enhancing the sensitivity of ovarian cancer cells to DDP.

Although the present study revealed a novel downstream molecular mechanism underlying the apoptosis of ovarian cancer cells caused by propofol treatment in vitro, further investigations are required to deeply understand this complex mechanism. However, in vivo systems are more complex and often provide more uncertainty than in vitro models; thus, whether this mechanism could be applied to an animal model is unknown. Therefore, in the future, this novel molecular mechanism will require further verification in more cell lines and in vivo mice models to prove its potential as a therapeutic target. 
In conclusion, the present study demonstrated that miR-374a negatively regulated FOXO1 expression to promote the proliferation and inhibit the apoptosis of ovarian cancer cells. Propofol decreased miR-374a expression to subsequently enhance FOXO1 expression and inhibit the proliferation of ovarian cancer cells, thus improving the sensitivity of ovarian cancer cells to DDP. This provides a theoretical basis for the adjuvant treatment of ovarian tumors with propofol.

\section{Acknowledgements}

Not applicable.

\section{Funding}

No funding was received.

\section{Availability of data and materials}

The datasets used and/or analyzed during the present study are available from the author on reasonable request.

\section{Authors' contributions}

YS and ZC conceived and designed the study, drafted the manuscript and revised the manuscript. YS, YP, LY, LM and $\mathrm{MZ}$ acquired analyzed and interpreted the data. All the authors approved publication of the final version and agree to be accountable for all aspects of the research in ensuring that the accuracy or integrity of any part of the work are appropriately investigated and resolved.

\section{Ethics approval and consent to participate}

Not applicable.

\section{Patient consent for publication}

Not applicable.

\section{Competing interests}

The authors declare that they have no competing interests.

\section{References}

1. Grunewald $\mathrm{T}$ and Ledermann JA: Targeted therapies for ovarian cancer. Best Pract Res Clin Obstet Gynaecol 41: 139-152, 2017.

2. Jaaback K, Johnson $\mathrm{N}$ and Lawrie TA: Intraperitoneal chemotherapy for the initial management of primary epithelial ovarian cancer. Cochrane Database Syst Rev 1: CD005340, 2016.

3. Zsiros E, Tanyi J, Balint K and Kandalaft LE: Immunotherapy for ovarian cancer: Recent advances and perspectives. Curr Opin Oncol 26: 492-500, 2014.

4. Shi C and Wang M: LINC01118 modulates paclitaxel resistance of epithelial ovarian cancer by regulating miR-134/ABCC1. Med Sci Monit 24: 8831-8839, 2018.

5. Papaevangelou E, Almeida GS, Box C, deSouza NM and Chung YL: The effect of FASN inhibition on the growth and metabolism of a cisplatin-resistant ovarian carcinoma model. Int J Cancer 143: 992-1002, 2018.

6. Chen SH and Chang JY: New insights into mechanisms of Cisplatin resistance: From tumor cell to microenvironment. Int J Mol Sci 20: E4136, 2019.
7. Jin P, Liu Y and Wang R: STAT3 regulated miR-216a promotes ovarian cancer proliferation and cisplatin resistance. Biosci Rep 38: BSR20180547, 2018.

8. Ai Z, Lu Y, Qiu S and Fan Z: Overcoming cisplatin resistance of ovarian cancer cells by targeting HIF-1-regulated cancer metabolism. Cancer Lett 373: 36-44, 2016.

9. Yang C, Gao J, Yan N, Wu B, Ren Y, Li H and Liang J: Propofol inhibits the growth and survival of gastric cancer cells in vitro through the upregulation of ING3. Oncol Rep 37: 587-593, 2017.

10. Yang N, Liang Y, Yang P, Yang T and Jiang L: Propofol inhibits lung cancer cell viability and induces cell apoptosis by upregulating microRNA-486 expression. Braz J Med Biol Res 50: e5794, 2017.

11. Chen X, Li K and Zhao G: Propofol inhibits HeLa cells by impairing autophagic flux via AMP-activated protein kinase (AMPK) activation and endoplasmic reticulum stress regulated by calcium. Med Sci Monit 24: 2339-2349, 2018.

12. Yu B, Gao W, Zhou H, Miao X, Chang Y, Wang L, Xu M and Ni G: Propofol induces apoptosis of breast cancer cells by downregulation of miR-24 signal pathway. Cancer Biomark 21: 513-519, 2018.

13. Wang P, Chen J, Mu LH, Du QH, Niu XH and Zhang MY: Propofol inhibits invasion and enhances paclitaxel-induced apoptosis in ovarian cancer cells through the suppression of the transcription factor slug. Eur Rev Med Pharmacol Sci 17: 1722-1729, 2013.

14. Kumar S, Boon RA, Maegdefessel L, Dimmeler S and Jo H: Role of noncoding RNAs in the pathogenesis of abdominal aortic aneurysm. Circ Res 124: 619-630, 2019.

15. Liu B, Li J and Cairns MJ: Identifying miRNAs, targets and functions. Brief Bioinform 15: 1-19, 2014.

16. Ma JB, Hu SL, Zang RK, Su Y, Liang YC and Wang Y: MicroRNA-487a promotes proliferation of esophageal cancer cells by inhibiting p62 expression. Eur Rev Med Pharmacol Sci 23: 1502-1512, 2019 .

17. He H, Zhao X, Zhu Z, Du L, Chen E, Liu S, Li Q, Dong J, Yang J and Lei L: MicroRNA-3191 promotes migration and invasion by downregulating TGFBR2 in colorectal cancer. J Biochem Mol Toxicol: e22308, 2019 doi: 10.1002/jbt.22308 (Epub ahead of print).

18. Wu H, Liu Y, Shu XO and Cai Q: MiR-374a suppresses lung adenocarcinoma cell proliferation and invasion by targeting TGFA gene expression. Carcinogenesis 37: 567-575, 2016.

19. Goto T, Takano M. Hirata J and Tsuda $\mathrm{H}$ : The involvement of FOXO1 in cytotoxic stress and drug-resistance induced by paclitaxel in ovarian cancers. Br J Cancer 98: 1068-1075, 2008.

20. Wang J, Yang H, Li W, Xu H, Yang X and Gan L: Thioredoxin 1 upregulates FOXO1 transcriptional activity in drug resistance in ovarian cancer cells. Biochim Biophys Acta 1852: 395-405, 2015.

21. Li N, Yang L, Wang H, Yi T, Jia X, Chen C and Xu P: MiR-130a and MiR-374a function as novel regulators of cisplatin resistance in human ovarian cancer A2780 cells. PLoS One 10: e0128886, 2015.

22. Livak KJ and Schmittgen TD: Analysis of relative gene expression data using real-time quantitative PCR and the 2(-Delta Delta C(T)) method. Methods 25: 402-408, 2001.

23. Huang L, Hu C, Chao H, Wang R, Lu H, Li H and Chen $\mathrm{H}$ : miR-29c regulates resistance to paclitaxel in nasopharyngeal cancer by targeting ITGB1. Exp Cell Res 378: 1-10, 2019.

24. Yue ZX, Gao RQ, Gao C, Liu SG, Zhao XX, Xing TY, Niu J, Li ZG, Zheng HY and Ding W: The prognostic potential of coilin in association with p27 expression in pediatric acute lymphoblastic leukemia for disease relapse. Cancer Cell Int 18: 106, 2018

25. Ajabnoor GM, Crook T and Coley HM: Paclitaxel resistance is associated with switch from apoptotic to autophagic cell death in MCF-7 breast cancer cells. Cell Death Dis 3: e260, 2012.

26. Gong S, Chen Y, Meng F, Zhang Y, Wu H, Li C and Zhang G: RCC2, a regulator of the RalA signaling pathway, is identified as a novel therapeutic target in cisplatin-resistant ovarian cancer. FASEB J 33: 5350-5365, 2019.

27. Li X, Zou Z, Tang J, Zheng Y, Liu Y, Luo Y, Liu Q and Wang Y: NOS1 upregulates ABCG2 expression contributing to DDP chemoresistance in ovarian cancer cells. Oncol Lett 17: 1595-1602, 2019

28. Norouzi-Barough L, Sarookhani MR, Sharifi M,Moghbelinejad S, Jangjoo S and Salehi R: Molecular mechanisms of drug resistance in ovarian cancer. J Cell Physiol 233: 4546-4562, 2018.

29. Chidambaran V, Costandi A and D'Mello A: Propofol: A review of its role in pediatric anesthesia and sedation. CNS Drugs 29: $543-563,2015$. 
30. Fan W, Zhu X, Wu L, Wu Z, Li D, Huang F and He H: Propofol: An anesthetic possessing neuroprotective effects. Eur Rev Med Pharmacol Sci 19: 1520-1529, 2015.

31. Zhang D, Zhou XH, Zhang J, Zhou YX, Ying J, Wu GQ and Qian JH: Propofol promotes cell apoptosis via inhibiting HOTAIR mediated mTOR pathway in cervical cancer. Biochem Biophys Res Commun 468: 561-567, 2015.

32. Liu SQ, Zhang JL, Li ZW, Hu ZH, Liu Z and Li Y: Propofol inhibits proliferation, migration, invasion and promotes apoptosis through down-regulating miR-374a in hepatocarcinoma cell lines. Cell Physiol Biochem 49: 2099-2110, 2018.

33. Li H, Lu Y, Pang Y, Li M, Cheng X and Chen J: Propofol enhances the cisplatin-induced apoptosis on cervical cancer cells via EGFR/JAK2/STAT3 pathway. Biomed Pharmacother 86: 324-333, 2017

34. Qian J, Shen S, Chen W and Chen N: Propofol reversed hypoxia-induced docetaxel resistance in prostate cancer cells by preventing epithelial-mesenchymal transition by inhibiting hypoxia-inducible factor $1 \alpha$. Biomed Res Int 2018: 4174232, 2018

35. Huang X, Teng Y, Yang H and Ma J: Propofol inhibits invasion and growth of ovarian cancer cells via regulating miR-9/NF- $\kappa \mathrm{B}$ signal. Braz J Med Biol Res 49: e5717, 2016.

36. Dasari S and Tchounwou PB: Cisplatin in cancer therapy: Molecular mechanisms of action. Eur J Pharmacol 740: 364-378, 2014.

37. Zhao C, Lu E, Hu X, Cheng H, Zhang JA and Zhu X: S100A9 regulates cisplatin chemosensitivity of squamous cervical cancer cells and related mechanism. Cancer Manag Res 10: 3753-3764, 2018.

38. He W, Feng L, Xia D and Han N: MiR-374a promotes the proliferation of human osteosarcoma by downregulating FOXO1 expression. Int J Clin Exp Med 8: 3482-3489, 2015.

39. Xu Y, Bei Y, Shen S, Zhang J, Lu Y, Xiao J and Li X: MicroRNA-222 promotes the proliferation of pulmonary arterial smooth muscle cells by targeting P27 and TIMP3. Cell Physio Biochem 43: 282-292, 2017.
40. Roy A and Banerjee S: p27 and leukemia: Cell cycle and beyond. J Cell Physiol 230: 504-509, 2015.

41. Li Y, Deng S, Peng J, Wang X, Essandoh K, Mu X, Peng T, Meng ZX and Fan GC: MicroRNA-223 is essential for maintaining functional $\beta$-cell mass during diabetes through inhibiting both FOXO1 and SOX6 pathways. J Biol Chem 294: 10438-10448, 2019.

42. Suzuki R, Amatya VJ, Kushitani K, Kai Y, Kambara T and Takeshima Y: MiR-182 and miR-183 promote cell proliferation and invasion by targeting FOXO1 in mesothelioma. Front Oncol 8: 446, 2018.

43. Li Y, Deutzmann A and Felsher DW: BIM-mediated apoptosis and oncogene addiction. Aging (Albany NY) 8: 1834-1835, 2016.

44. Li L, Wang C, Wen Y, Hu Y, Xie Y, Xu M, Liang M, Liu W, Liu L and $\mathrm{Wu} \mathrm{Y}$ : ERK1/2 and the Bcl-2 family proteins Mcl-1, tBid, and Bim are involved in inhibition of apoptosis during persistent chlamydia psittaci infection. Inflammation 41: 1372-1383, 2018.

45. Huang C, Chen D, Zhu H, Lv S, Li Q and Li G: LITAF enhances radiosensitivity of human glioma cells via the FoxO1 pathway. Cell Mol Neurobiol 39: 871-882, 2019.

46. Qin Y, Li L, Wang F, Zhou X, Liu Y, Yin Y and Qi X: Knockdown of Mir-135b sensitizes colorectal cancer cells to oxaliplatin-induced apoptosis through increase of FOXO1. Cell Physiol Biochem 48: 1628-1637, 2018.

This work is licensed under a Creative Commons Attribution-NonCommercial-NoDerivatives 4.0 International (CC BY-NC-ND 4.0) License. 